\title{
Localized spectral analysis of global satellite gravity fields for recovering time-variable mass redistributions
}

\author{
Shin-Chan Han • Pavel Ditmar
}

Received: 3 April 2007 / Accepted: 31 October 2007 / Published online: 23 November 2007

(C) Springer-Verlag 2007

\begin{abstract}
A spatiospectral localization method is discussed for processing the global geopotential coefficients from satellite mission data to investigate time-variable gravity. The time-variable mass variation signal usually appears associated with a particular geographical area yielding inherently regional structure, while the dependence of the satellite gravity errors on a geographical region is not so evident. The proposed localization amplifies the signal-to-noise ratio of the (non-stationary) time-variable signals in the geopotential coefficient estimates by localizing the global coefficients to the area where the signal is expected to be largest. The results based on localization of the global satellite gravity coefficients such as Gravity Recovery And Climate Experiment (GRACE) and Gravity and Ocean Circulation Explorer (GOCE) indicate that the coseismic deformation caused by great earthquakes such as the 2004 Sumatra-Andaman earthquake can be detected by the low-low tracking and the gradiometer data within the bandwidths of spherical degrees 15-30 and 25-100, respectively. However, the detection of terrestrial water storage variation by GOCE gradiometer is equivocal even after localization.
\end{abstract}

\footnotetext{
S.-C. Han $(\varangle)$

Planetary Geodynamics Laboratory, Code 698,

NASA Goddard Space Flight Center, Greenbelt, MD 20771, USA

e-mail: schan@puuoo.gsfc.nasa.gov

S.-C. Han

Goddard Earth Science and Technology Center, University of Maryland Baltimore County,

Baltimore, MD 21228, USA

P. Ditmar

Department of Earth Observation and Space systems (DEOS), Faculty of Aerospace Engineering, Delft University of Technology, Kluyverweg 1, 2629 HS Delft, The Netherlands

e-mail:ditmar@geo.tudelft.nl
}

Keywords Spatiospectral localization . Spherical harmonic analysis · GRACE - GOCE . Hydrology · Earthquake

\section{Introduction}

Recently there has been an increasing number of geophysical applications from satellite gravity measurements. Monthly time-series of the Earth gravity field from the Gravity Recovery And Climate Experiment (GRACE) data, in the form of spherical harmonic coefficients, have been producing indispensable implications and insights about large scale terrestrial water, ocean, ice sheet, fast and slow deformations of the solid Earth such as earthquake and post-glacial rebound (e.g., Tapley et al. 2004b; Chambers et al. 2004; Luthcke et al. 2006; Han et al. 2006; Tamisiea et al. 2007).

These temporal mass variations observed by the GRACE satellites are limited mostly to low degree and order harmonics (e.g., less than 20 or so) of the monthly gravity solutions due to limitation of current capability of the intersatellite ranging instrument and to errors in applied geophysical models (Wahr et al. 2006). Undesired errors in the global harmonic coefficient estimates (mostly higher degrees and orders), associated primarily with measurement noise and aliasing effect from un- and mis-modeled geophysical signals such as tides and atmosphere (Han et al. 2004), are reduced in somewhat empirical ways by applying filtering methods to the estimated coefficients or smoothing in space domain (Davis et al. 2004; Han et al. 2005; Wahr et al. 2006; Swenson and Wahr 2006; Kusche 2007).

It is a degree variance (or its square root, degree-RMS) that has been often used to quantify the powers of signal and error in the gravity field estimates at various spatial wavelengths (Kaula 1967). In addition to admittance and 
correlation functions, it is useful to analyze and compare the global topography and (static) gravity to understand physical implications of their spatial variations for Earth and other planets' geodynamics (e.g., Kaula 1967; Wieczorek 2007).

A direct spectral power comparison (through degree variance) of a certain time-variable mass signal and global gravity coefficients estimated from satellite data, however, should be avoided because the time-variable signal (such as earthquake and hydrology) arises largely associated with a confined geographical regime and decay rapidly away from the region while satellite gravity data contain all other signals and errors outside the region of interest. That is, the time-variable signal of interest is non-stationary while the satellite gravity error is more likely to be stationary. Therefore the comparison between them would yield incorrect signal-to-noise ratio (SNR) for a particular (locally intense) signal by globally averaging and thus underestimating the non-stationary time-variable signal. Although we observe that the power of some historical great earthquake deformation signals are one or two order of magnitude smaller compared to GRACE's actual accuracy (Sun and Okubo 2004; Gross and Chao 2001), the 2004 Sumatra-Andaman earthquake deformation signal was successfully detected by localized analysis of GRACE monthly harmonic coefficients (Han and Simons 2007) as well as satellite tracking data (Han et al. 2006).

In this paper, we present a new way to process the global spherical harmonic coefficients for time-variable mass variation by spatiospectral localization, which was originally used to study distinct local features in the (static) global gravity and topography data (Simons and Hager 1997; Simons et al. 1997). The spatiospectral localization of the global spherical harmonic fields to the area where the signal is expected to be largest will provide better quantification of the local signal from the global spherical harmonic fields. The way of processing the satellite gravity estimates is fundamentally different from the ones based on spatial smoothing over the globe that has been used widely in the community. Finally, we demonstrate potential to detect the earthquake deformation from a new satellite mission, Gravity and Ocean Circulation Explorer (GOCE), carrying a space-borne gravity gradiometer (SGG).

\section{Global and localized signal-to-noise ratio}

The spectral power of the global gravity field at various spatial wavelengths on a sphere (i.e., degree variance or degree-RMS) is often calculated to quantify the variability of geophysical signals and the errors of the gravity fields derived from satellite data (e.g., Gross and Chao 2001). Figure 1 shows the error degree-RMS of actual GRACE monthly gravity field estimates (Tapley et al. 2004a; Wahr et al. 2006)

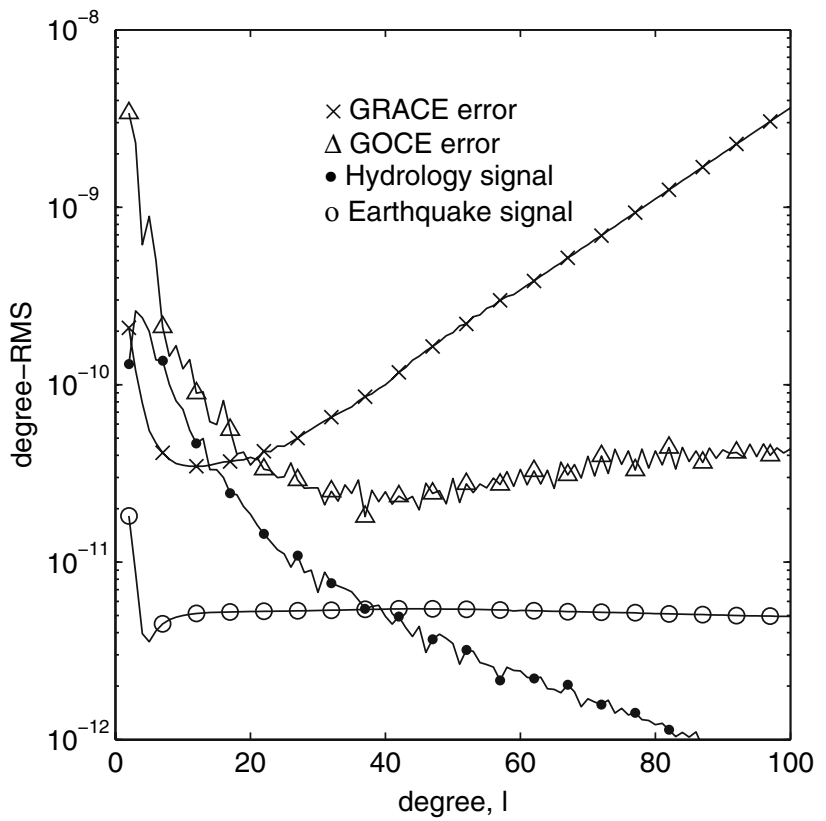

Fig. 1 Degree-RMS of the errors in the satellite gravity field estimates from GRACE (cross) and from GOCE SGG (triangle) and of the signals associated with terrestrial water storage (dot) and with the 2004 great Sumatran-Andaman earthquake (circle). The GRACE error indicates actual monthly accuracy attainable from the GRACE satellite tracking data and the GOCE error curve implies simulated accuracy expected from two months of the GOCE gradiometer observations. The variability caused by hydrology represents a monthly mean variation with respect to a year-average field. The earthquake signal indicates the coseismic variation before and after the event

and simulated GOCE SGG gravity field (ESA 1999; Ditmar et al. 2003). Figure 1 also presents the degree-RMS of two geophysical signals such as terrestrial water storage calculated by spherical harmonic decomposition of Land Data Assimilation System (LDAS) (Fan et al. 2003) and the 2004 great Sumatra-Andaman earthquake calculated by normal mode summation (Gross and Chao 2006).

The terrestrial water mass has greater power than GRACE gravity field error over the degrees less than 15 , indicating possible detection by GRACE gravity fields. However, the degree-RMS (globally calculated) shows that both satellite gravity estimates are too noisy to detect earthquake-induced gravity changes yielding more than one order of magnitude larger error at all frequencies, when compared to the signal. This has been a typical way to quantify the sensitivity of gravity fields to the geophysical signals (e.g., Gross and Chao 2001; Sun and Okubo 2004).

This comparison, however, disregards the distinct differences in spatial distribution of the signals and errors. While many of geophysical signals causing temporal change in the gravity fields are locally intense and highly non-stationary, the satellite gravity errors are relatively uniform over the globe. Naturally the power of geophysical signals may be stronger over a certain geographical region and rapidly 
decays outside the region, e.g., the hydrology signal within a specific river basin and earthquake deformation near the epicenter. On the other hand, the satellite gravity error, in principle, does not have such geographically dependent pattern except for the dependence on latitude. Therefore, it is readily expected that the SNR would depend on the geographical region because of the geographical dependence of the signal's power.

We can quantify the (localized) signal and error associated only with a specific region from the global spherical harmonic coefficients by applying a spatial window function centered at the region of interest (called spatiospectral localization) (Simons et al. 1997; Simons and Hager 1997; Wieczorek and Simons 2005). The following describes operational summary to explain how the localized spectra are computed. For more theoretical elaboration and details, the readers are recommended to refer to Simons et al. (1997) and Wieczorek and Simons $(2005,2007)$.

First, we consider the signal $s(\Omega)$ and the window function $h(\Omega)$ defined on a sphere and thus expressed into a series of (surface) spherical harmonic functions. The localized version of the signal, denoted by $y(\Omega)$, is computed simply by multiplying the signal and window in space domain such as $y(\Omega)=s(\Omega) h(\Omega)$. The (fully normalized) expansion coefficients of the localized signal, which we are interested in, can be computed by $\bar{C}_{l m}^{y}=\frac{1}{4 \pi} \int s(\Omega) h(\Omega) \bar{P}_{l m}(\cos \theta) \cos$ $(m \lambda) \mathrm{d} \Omega$ and $\bar{S}_{l m}^{y}=\frac{1}{4 \pi} \int s(\Omega) h(\Omega) \bar{P}_{l m}(\cos \theta) \sin (m \lambda) \mathrm{d} \Omega$, where $\Omega=(\theta, \lambda)$ and $\bar{P}_{l m}(\cos \theta)$ is the (fully normalized) associated Legendre function of degree $l$ and order $m$. Reminding that $s(\Omega)$ and $h(\Omega)$ are also expressed with the series of spherical harmonic functions, it can be imagined that those integrals essentially involve integrals of three spherical harmonic functions and multiplication of the signal and window spectra. The integral of three spherical harmonic functions can be calculated by use of Wigner- $3 \mathrm{j}$ functions as documented in Dahlen and Tromp (1998).

We often consider an isotropic window that can be expressed only with zonal spherical harmonic series. The computation of the harmonic coefficients of the localized version of the signal with the isotropic window is simplified after evaluating the integrals of triple products of spherical harmonic functions as follows (also given in Eq. (10) of Wieczorek and Simons 2005):

$$
\begin{aligned}
\bar{C}_{l m}^{y}= & \sum_{j=0}^{L_{h}} \sum_{i=|l-j|}^{l+j} h_{j} \bar{C}_{i m}^{s} \sqrt{(2 i+1)(2 j+1)(2 l+1)}(-1)^{m} \\
& \times\left(\begin{array}{ccc}
i & j & l \\
0 & 0 & 0
\end{array}\right)\left(\begin{array}{ccc}
i & j & l \\
m & 0 & -m
\end{array}\right)
\end{aligned}
$$

where $L_{h}$ is the maximum expansion degree of the window and $h_{j}$ is the expansion coefficients of the (isotropic) window (no order dependence). The last two parentheses are
Wigner-3j functions. $\bar{S}_{I m}^{y}$ is computed simply by replacing $\bar{C}_{l m}^{s}$ with $\bar{S}_{l m}^{s}$, where $\bar{C}_{l m}^{s}$ and $\bar{S}_{l m}^{s}$ are the coefficients of the signal $s(\Omega)$. Alternatively, the localized spectra of the signal can be computed numerically by spherical harmonic decomposition of the spatial map $s(\Omega) h(\Omega)$ shown in Driscoll and Healy (1994) for example.

As implied by (1), the coefficients of the localized (or windowed) signal are nothing but linear combination of the signal coefficients $\bar{C}_{l m}^{s}$ and $\bar{S}_{l m}^{s}$ with the weights defined by the choice of the window function. Unlike the ubiquitous spatial smoothing methods (convolution of various smoothing kernels with the data in space domain) applied for GRACE data processing introduced in many literatures (some of which are mentioned in the previous section), the localization is a type of spectral smoothing. That is, the localized coefficient of degree $l$ is computed with the original coefficients within the bandwidth of $\max \left(0, l-L_{h}\right) \leq l \leq l+L_{h}$. If the original signal is known only to a certain degree such as $L_{s}$, it can be readily seen from the upper bound of the summation in (1) that the permissible range of the localized coefficients is limited to $L_{s}-L_{h}$, meaning that the spatial resolution of the windowed field is decreased by an amount of the maximum degree of the window function. Also the windowed spectra of the low degrees $\left(l<L_{h}\right)$ can be biased significantly as noted in Sect. 5.1 of Wieczorek and Simons (2005). It is the reason why we need to have a compact representation of the window function by keeping the maximum expansion degree $L_{h}$ as small as possible but still keeping most of energy within the area of interest.

Section 3.1 in Wieczorek and Simons (2005) elaborated how to obtain the optimal window function considering the concentration ratio $\lambda$ of its energy within the area of focus that is given as

$\lambda=\int_{\Omega_{0}} h^{2}\left(\Omega^{\prime}\right) \mathrm{d} \Omega^{\prime} / \int_{\Omega} h^{2}\left(\Omega^{\prime}\right) \mathrm{d} \Omega^{\prime}$,

where $\Omega$ and $\Omega_{0}$ denote the spherical domains of the entire globe and of the spherical cap, respectively. The bandlimited window (isotropic) function $h$ is to be determined such that the concentration ratio $\lambda$ is maximized. One chooses the size of the window $\Omega_{0}$ and maximum degree $L_{h}$ of spherical harmonic expansion. It turns out that the eigenvector and eigenvalue of the matrix explicitly given in Eq. (13) of Wieczorek and Simons (2005) are the (Legendre) spectra of the window function and the concentration ratio, i.e., the solutions of the maximization problem.

In this study, we use two spherical cap window functions; the one with a spherical cap radius of $25^{\circ}$ that is expanded by spherical harmonic functions up to degree 15 and the other with a radius of $40^{\circ}$ and maximum degree of 10 . The first and second windows are designed for studying the 2004 great Sumatra-Andaman earthquake and the Amazon hydrology 


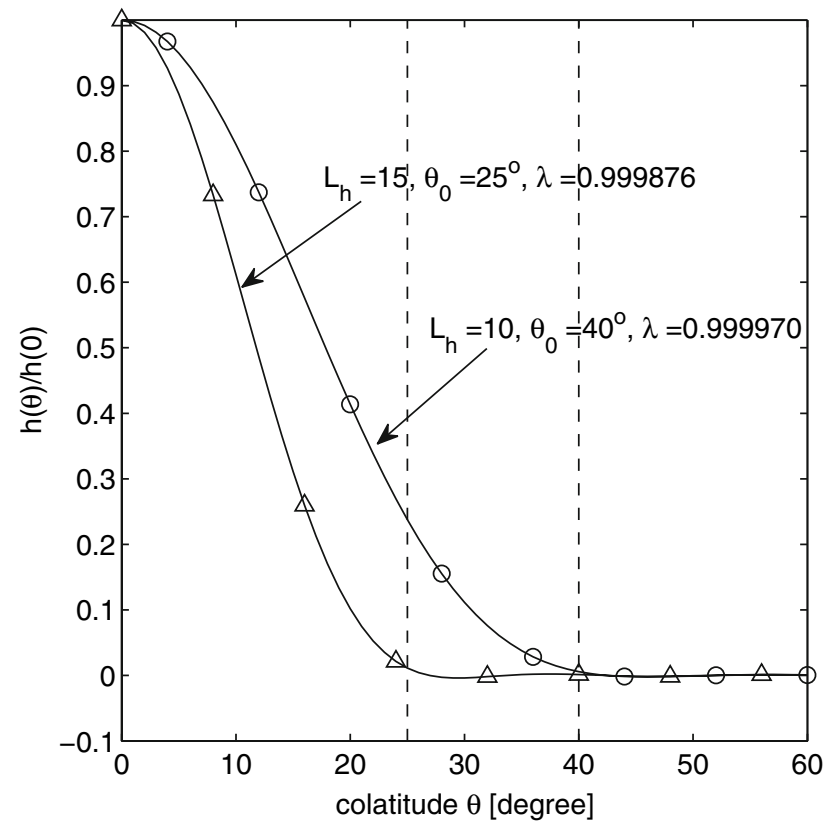

Fig. 2 Cross sections of the localizing window functions with various parameters such as maximum degree of expansion $L_{h}$, spherical cap size $\theta_{0}$, and concentration ratio $\lambda$. Each function is normalized vertically with the maximum value at $\theta=0^{\circ}$, and is depicted from $\theta=0^{\circ}$ to $\theta=60^{\circ}$

from the GRACE and GOCE gravity fields, respectively, guaranteeing enough spatial coverage for those signals. The maximum degree $L_{h}$ is determined by considering Shannon number $N_{0}$ that is given as $\left(L_{h}+1\right) \theta_{0} / \pi$ (Wieczorek and Simons 2005). Typically $N_{0}-1$ gives the number of wellconcentrated windows. We use a single most-concentrated window by keeping $N_{0}$ close to 2 .

Figure 2 shows the cross section of the two window functions over the spherical distance from the center. Both cases are band-limited and thus global-support but optimal in the sense that the energy is maximally concentrated within the pre-defined spherical cap centered to the region of interest. The concentration ratio for the first window exceeds 0.999876 and the one of the second window does 0.999970 , which means that less than $0.1 \%$ of the identified signal and error would originate from outside the region of interest with both windows. The plot is normalized vertically by the maximum value $h(0)$. There are oscillations of the windows beyond the spherical cap radius $\theta_{0}$, but the power when $\theta>\theta_{0}$ is considerably smaller ( 6 orders of magnitude smaller) than the power when $\theta \leq \theta_{0}$ producing negligible contribution of the data outside the cap to the windowed field. Considering the size of the concentration region of each window, the first and second windows $\left(\theta_{0}=25^{\circ}\right.$ and $\theta_{0}=40^{\circ}$ ) are used for studying the 2004 SumatraAndaman earthquake and the Amazon hydrology, respectively, from the global satellite gravity fields.
We show how localization enhances the sensitivity of satellite gravity data to the geophysical signals by quantifying the SNR before and after localization. The degree-RMS (square root of power) of the band-limited signal up to $L_{S}$ is given as follows:

$V^{s}(l)=\sqrt{\sum_{m=0}^{l}\left(\bar{C}_{l m}^{s}\right)^{2}+\left(\bar{S}_{l m}^{s}\right)^{2}}, \quad 0 \leq l \leq L_{s}$.

The error degree-RMS is often available from the satellite mission analysis and has been commonly used to quantify the accuracy of the gravity field estimates at various wavelengths. It is defined as

$$
V^{e}(l)=\sqrt{\sum_{m=0}^{l}\left(\left\langle\bar{C}_{l m}^{e}\right\rangle\right)^{2}+\left(\left\langle\bar{S}_{l m}^{e}\right\rangle\right)^{2}}, \quad 2 \leq l \leq L_{s},
$$

where \langle\rangle is the expectation operator. There is no consideration of correlations among the errors in various degrees and orders. We define the SNR as follows:

$Z_{\text {global }}(l)=V^{s}(l) / V^{e}(l), \quad 2 \leq l \leq L_{s}$.

As implied by (5), we are interested in wavelength-dependent SNR of geophysical signals and satellite gravity field errors. Figure 3 presents the 4 combinations of SNR including mean hydrology (LDAS) and earthquake (e.g., December 2004 Sumatra-Andaman) signals and GRACE and GOCE gravity field errors, as used in Fig. 1. The hydrology signal in the GRACE gravity estimates is expected to yield SNR greater than unity when $l \leq 15$. Both hydrology and earthquake signals show SNR less than unity at all wavelengths from the GOCE gravity estimates, indicating the gravity estimates are too noisy to recovery those signals. Likewise, GRACE gravity seems to be too noisy to detect the earthquake signal according to this global spectra comparison.

We examine the SNR after localization of the geophysical signals and the satellite gravity fields. In a similar way, the degree-RMS of the localized signals are computed as follows:

$$
V^{y}(l)=\sqrt{\sum_{m=0}^{l}\left(\bar{C}_{l m}^{y}\right)^{2}+\left(\bar{S}_{l m}^{y}\right)^{2}}, \quad L_{h} \leq l \leq L_{s}-L_{h} .
$$

Note that the localized coefficients (smoothed spectra), $\bar{C}_{l m}^{y}$ and $\bar{S}_{l m}^{y}$, are computed from degree $L_{h}$ to $L_{s}-L_{h}$ using (1). The expectation of the localized error degree-RMS can be computed on the basis of the degree-RMS estimates of stationary error $V^{e}(l)$ introduced in (4). The derivation in Appendix C of Wieczorek and Simons (2005) shows that it 


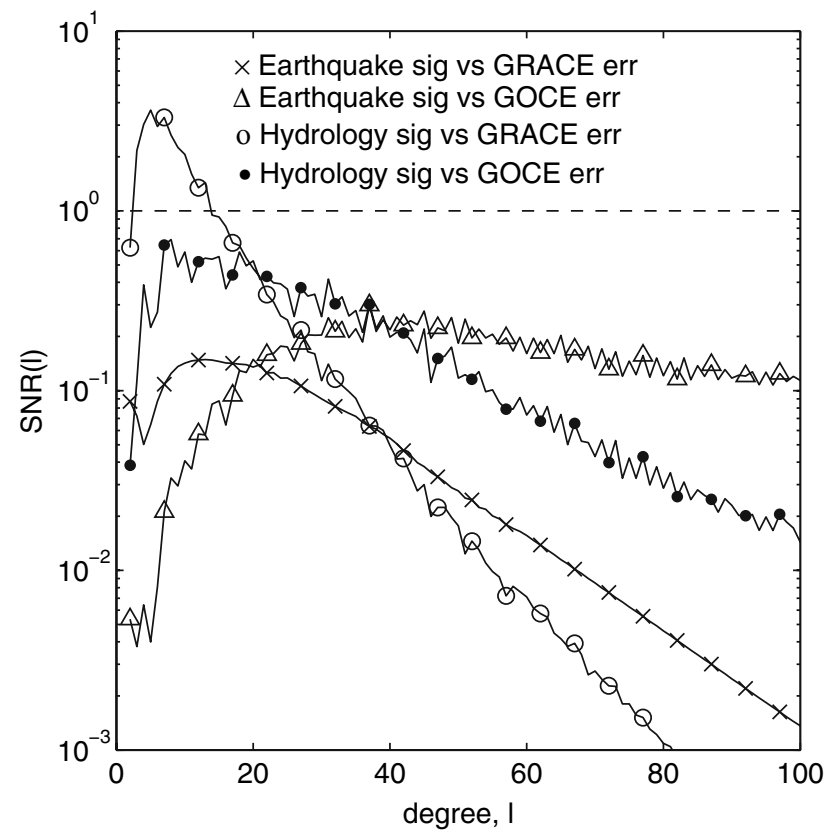

Fig. 3 Signal to noise ratio (SNR) between either earthquake or hydrology signal and either GRACE or GOCE gravity field estimate error. SNR is depicted with various wavelengths $(40,000 \mathrm{~km} / l)$ or spherical harmonic degrees. Degree-RMS shown in Fig. 1 has been used to compute this degree-dependent SNR. Note that the SNR is less than unity except the hydrology signal at low degrees $(l \leq 15)$ of the GRACE gravity field

is explicitly given by as follows:

$$
\begin{aligned}
& V^{\varepsilon}(l)= \sqrt{(2 l+1) \sum_{j=0}^{L_{h}} h_{j}^{2} \sum_{i=|l-j|}^{l+j}\left(V^{e}(i)\right)^{2}\left(\begin{array}{lll}
i & j & l \\
0 & 0 & 0
\end{array}\right)^{2}}, \\
& L_{h} \leq l \leq L_{s}-L_{h} .
\end{aligned}
$$

Finally, the localized SNR is computed by taking the ratio of (6) and (7) as follows:

$$
Z_{\text {local }}(l)=V^{y}(l) / V^{\varepsilon}(l), \quad L_{h} \leq l \leq L_{s}-L_{h} .
$$

The spatial windowing may suppress significantly the signals and errors outside the region of focus and makes the localized coefficients retain the signals and errors originated only from the region where the spatial window is applied. The signals are strong within the region and decay quite rapidly away from the region while the errors are stationary over the globe. Consequently, the windowing applied at the region enhances SNR.

Figure 4 presents the same as Fig. 3 but after applying the spatiospectral localization. All SNR have been improved such that the earthquake signal is detectable from the localized gravity coefficients estimated from both satellite missions, GRACE and GOCE. The coseismic signal, when localized near the epicenter, shows powers stronger than GRACE gravity error within the bandwidth from degree

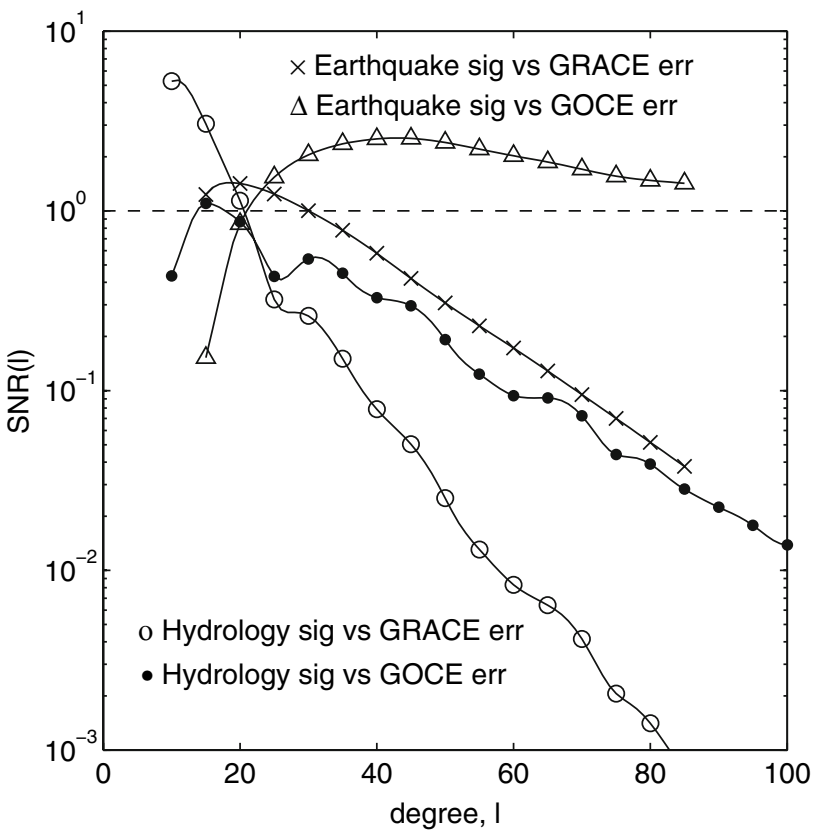

Fig. 4 The same as Fig. 3 but after applying the spatiospectral localization to both the geophysical signals and GRACE and GOCE gravity error spectra. Note that the localization increases SNR by suppressing the errors outside the region of interest from the global satellite gravity field estimates without much suppression of the signal. All SNR's have been significantly increased yielding SNR greater than unity for the earthquake signal from both satellite gravity fields. Especially, GOCE gravity field is predicted to recover the earthquake signal with enhanced resolution to degree 100 or so. For the 2004 Sumatra-Andaman earthquake and Amazon hydrology signals, the windows with the spherical cap size of $\theta_{0}=25^{\circ}$ and $\theta_{0}=40^{\circ}$ have been used, respectively

15 to 30. Han and Simons (2007) analyzed 45 monthly timeseries of GRACE gravity fields and reported significant jumps (coseismic changes) caused by the earthquake in the timeseries of the individual coefficients within the bandwidth after localization.

More interestingly, the earthquake signal would be greater than GOCE gravity error after localization from degree 20 to quite higher degrees up to 100 or so (Note that Fig. 4 shows SNR to degree 85 since the synthetic earthquake data are expanded to degree 100 and the applied window is expanded to degree 15). This is likely due to the sensitivity of the gradiometer to higher degree and order harmonics and the locally intense nature of the earthquake-induced signal spreading its power to higher degrees. However, the detection of hydrology signal from GOCE is still ambiguous which is implied by the SNR close to or slightly less than unity only within the quite narrow bandwidth from 15 to 20 .

\section{Simulation for space-borne gradiometer mission GOCE}

To verify possible detection of the earthquake signal from the GOCE SGG gravity fields foreseen by the localized 
sensitivity analysis, we have conducted an end-to-end simulation of the GOCE gravity field recovery considering plausible noise of the gradiometer instrument (ESA 1999; Alenia 2001; Ditmar and Klees 2002). We generated the gravity gradient tensor anomalies induced by the coseismic deformation of the 2004 Sumatra-Andaman earthquake (expanded to degree and order 100) along the GOCE satellite orbits in a Sun-synchronous near-polar orbit ( $97^{\circ}$ of inclination) and at orbital altitude of $250 \mathrm{~km}$ for two months every 5 -s interval. We have estimated the spherical harmonic coefficients up to degree and order 300 using two months of synthetically calculated gravity gradient tensor components including the colored noise of the gradiometer and the earthquake-induced signals. The earthquake perturbation of the gravity gradient tensor at the GOCE altitude is less than $\pm 1 \mathrm{mE}$ (largest in the main-diagonal radial component).

The two sets of global harmonic solutions have been estimated by inverting 2 months worth of synthetic gradiometer observations with and without the earthquake-induced perturbation, but under the colored noise for both cases, using the independently developed software system in Ditmar et al. (2003). The GOCE gravity solutions have been localized with the window shown in Fig. 2 (the one with spherical cap size of $\theta_{0}=25^{\circ}$ ). We tested three localized coefficients of the GOCE estimates including (1) the coefficient estimates from the noisy gradiometer data perturbed by the earthquake and localized at the epicenter; (2) the same as (1) but localized around the location $180^{\circ}$ separated from the epicenter; and (3) the ones estimated from the noisy gradiometer data excluding the earthquake signal and localized at the epicenter. In principle, the case (2) should contain the earthquake signal but the magnitude should be significantly reduced because the signal attenuates rapidly from the epicenter. Therefore, the power spectrum of the case (2) is expected to be comparable to the one of the case (3) since the earthquake signal is negligible at the distant location and the gravity error is stationary. Finally we localize the earthquake model coefficients at the epicenter for comparison and call them the case (4).

Figure 5 shows various degree-RMS curves of the above cases (see also the description in the caption). The degreeRMS comparisons of the cases between (1) and (3) and between (1) and (2) indicate that there are significant powers associated with the earthquake within the bandwidth between degree 25 and 100 and they appear when localized only around the epicenter. The amount of the extra power follows the power of the localized earthquake signal (case (4)) within the bandwidth $(25 \leq l \leq 85)$ remarkably well. It indicates that the GOCE spherical harmonic coefficient estimates may reveal the earthquake signal within the band only after localization at the right place (i.e., epicenter).

We also computed the corresponding gravity changes in spatial domain from the localized GOCE gravity solution, but

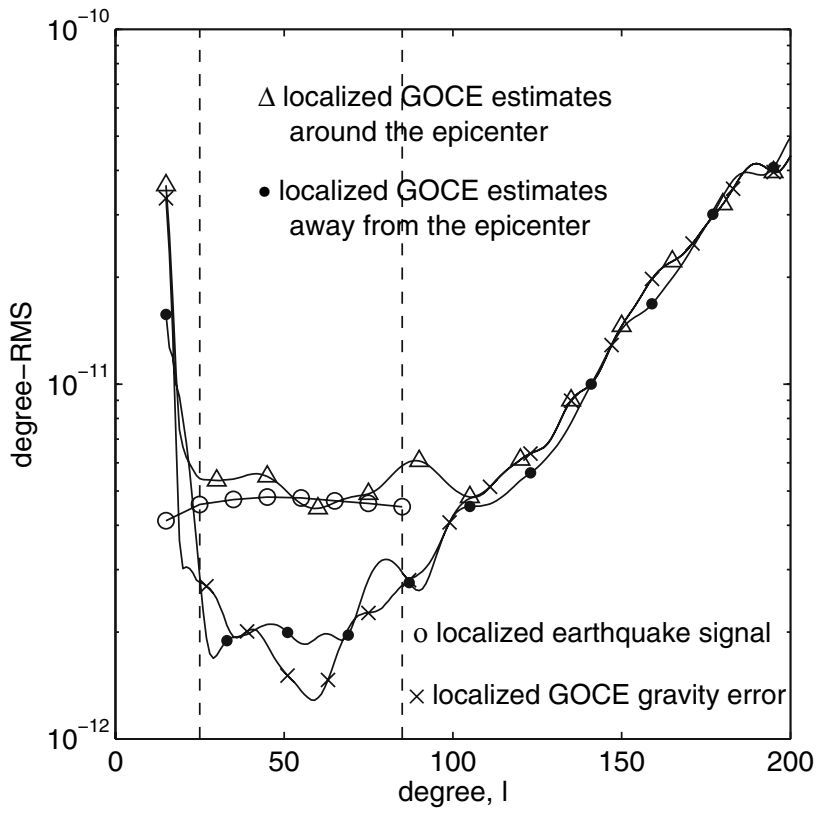

Fig. 5 Degree-RMS of the following coefficients after applying the localization window (cap size of $\theta_{0}=25^{\circ}$ ): the case (1) - GOCE gravity coefficients estimated from the gradiometer observations including the synthetic earthquake signal and instrument noise and localized at the epicenter (triangle), the case (2) - the same as (1) but localized at the place $180^{\circ}$ away from the epicenter (dot), the case (3)-GOCE gravity estimate from two months of gradiometer observations under plausible colored noise only and localized at the epicenter (cross), and finally the case (4) — synthetic earthquake signal expanded to degree and order 100 and localized at the epicenter (circle)

only from the band-limited spectra (degree 25-85). Figure 6 shows the gravity changes from the localized coefficients such as (a) GOCE estimates from the observations including the synthetic earthquake signal and instrument noise; (b) the earthquake model used in the simulation; (c) the same as (a) but from the coefficient estimates using the measurements excluding the synthetic earthquake perturbation, indicating merely observational noise (after localization); and (d) the same as (a) but using the localization window centered at the place $180^{\circ}$ away from the epicenter. Note that the magnitudes of (c) and (d) are quite similar indicating that the earthquake perturbation is significantly reduced away from the epicenter and that they are mostly due to the gradiometer instrument noise. The recovery from the localized GOCE estimates is in good agreement with the reference model varying more than $\pm 100 \mu \mathrm{Gal}$ and the deviation from the truth model is about a couple of tens $\mu \mathrm{Gal}$ which is well within the measurement error level after localization.

\section{Remark}

We discussed a way to quantify the time-variable gravity signals associated only with a particular region by localizing the 

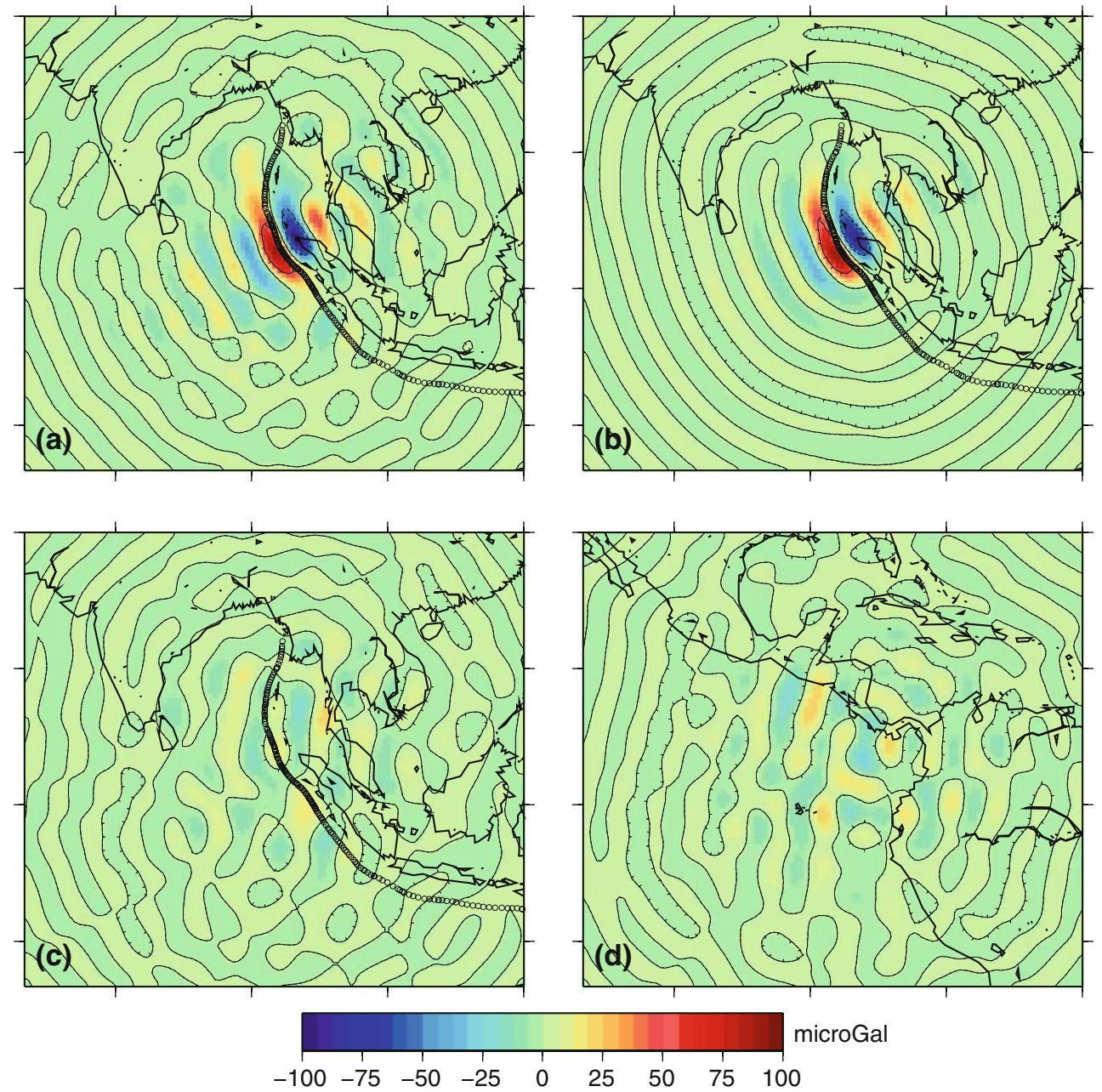

Fig. 6 (a), (b), (c), and (d) present gravity changes computed from the localized coefficients corresponding to the cases (1), (4), (3), and (2) of Fig. 5, respectively. Note that only band-limited spectra $(25 \leq l \leq 85)$ yielding the signal-to-noise ratio greater than unity have been used to compute the gravity change

global geopotential coefficients at the place where majority of the signals originate. The most outstanding effect of the localization is to improve the SNR of the global gravity solutions. The localization also decreases the spatial resolution of the fields by the amount of the maximum expansion degree of the window function and reduces the signal itself.

However, those undesired effects are comparatively much less than the benefits obtained by suppressing the errors in the original fields yielding improved SNR over the particular geographical region. The localized degree-RMS indicates that the 2004 Sumatra-Andaman earthquake is greater than the GRACE error within the band from degree $\sim 15$ to $\sim 30$. It has been predicted that such earthquake can be detected from GOCE SGG with enhanced spatial resolution (bandwidth from degree 25 to 100). An independent end-to-end simulation for the GOCE gravity field recovery on the basis of two months of gradiometer measurements verified the possible detection predicted from localized power spectrum.
A single most-concentrated window has been used in this study while the analysis based on multiple windows (all are concentrated within the region of interest with various concentration ratios) is possible. Although the single window is shown to be quite effective to retrieve the time-variable signals and may be superior to other spatial smoothing approach, the multiple windows may be useful to extract the local signal even better. It is due to the fact that the energy of the multiple windows is more uniformly distributed and thus represent the region of interest better. Also the effects of various parameterizations such as choices of expansion degree and spatial size of windows have not been investigated. This study reserves the rooms for future investigation about the optimal selections of window parameters and the enhancement by using multiple windows.

Acknowledgements This work was supported by the U.S. National Aeronautical and Space Administration GRACE project. We acknowledge the NASA/GFZ GRACE project for the GRACE data products 
distributed by Physical Oceanography Distributed Active Archive Center. We thank Mark Wieczorek for providing the FORTRAN codes for this work via www.ipgp.jussieu.fr/ wieczor/SHTOOLS, Richard Gross for providing the global spherical harmonic expansion coefficients of the 2004 great Sumatra-Andaman earthquake signal, and Frederik Simons for valuable discussions. We also thank Jürgen Kusche, Nico Sneeuw, Will Featherstone, and two anonymous reviewers for comments to help clarify many things in this paper. Realizations of random frequency-dependent noise were generated by Roland Klees. The realistic GOCE orbit was computed by E.J.O. Schrama. Computing resource was provided partially by Stichting Nationale Computerfaciliteiten grant SG-027.

\section{References}

Alenia (2001) Performance requirements and budgets for the gradiometric mission. Technical note G0-TN-AI-0027, Turin, Italy

Chambers D, Wahr J, Nerem R (2004) Preliminary observations of global ocean mass with GRACE, Geophys Res Lett 31:L13310. doi:10.1029/2004GL020461

Dahlen FA, Tromp J (1998) Theoretical global seismology. Princeton University Press, Princeton

Davis JL, Elosegui P, Mitrovica JX, Tamisiea ME (2004) Climate-driven deformation of the solid Earth from GRACE and GPS. Geophys Res Lett 31: L24605. doi:10.1029/2004GL021435

Ditmar P, Klees R (2002) A method to compute the Earth's gravity field from SGG/SST data to be acquired by the GOCE satellite. Delft University Press

Ditmar P, Klees R, Kostenko F (2003) Fast and accurate computation of spherical harmonic coefficients from satellite gravity gradiometry data. J Geod 76:690-705

Driscoll JR, Healy DM (1994) Computing Fourier transforms and convolutions on the 2-sphere. Adv Appl Math 15:202-250

ESA (1999) Gravity field and steady-state ocean circulation mission. Rep. SP-1233, European Space Agency, Noordwijk

Fan Y, andel Dool H, Mitchell K, Lohmann D (2003) A 51-year reanalysis of the U.S. land-Surface hydrology. GEWEX News 13: 6-10

Gross RS, Chao BF (2001) The gravitational signature of earthquakes. In: Gravity, geoid and geodynamics: GGG2000 IAG international symposium, 2000, vol 123, pp 205-210. Springer, New York

Gross RS, Chao BF (2006) The rotational and gravitational signature of the December 26, 2004 Sumatran earthquake. Surv Geophy 27(6): 615-632

Han SC, Jekeli C, Shum CK (2004) Time-variable aliasing effects of ocean tides, atmosphere, and continental water mass on monthly mean GRACE gravity field. J Geophys Res 109:B04403. doi:10.1029/2003JB002501

Han SC, Shum CK, Jekeli C, Kuo C, Wilson CR, Seo KW (2005) Nonisotropic filtering of GRACE temporal gravity for geophysical signal enhancement. Geophy J Int 163: 18-25
Han SC, Shum CK, Bevis MG, Ji C, Kuo C (2006) Crustal dilatation observed by GRACE after the 2004 Sumatra-Andaman earthquake. Science 313: 658-662

Han SC, Simons FJ (2007) Spatiospectral localization of global geopotential fields from GRACE reveals the coseismic gravity change due to the 2004 Sumatra-Andaman earthquake. J Geophys Res (in-press)

Kaula WM (1967) Geophysical implications of satellite determinations of the Earth's gravitational field. Space Sci Rev 7: 769-794

Kusche J (2007) Approximate decorrelation and non-isotropic smoothing of time-variable GRACE-type gravity field models. J Geod (in-press)

Luthcke SB, Zwally HJ, Abdalati W, Rowlands DD, Ray RD, Nerem RS, Lemoine FG, McCarthy JJ, Chinn DS (2006) Recent Greenland ice mass loss by drainage system from satellite gravity observations. science 314:1286-1289. doi:10.1126/science.1130776

Simons M, Solomon S, Hager B (1997) Localization of gravity and topography: constraints on the tectonics and mantle dynamics of Venus. Geophys J Int 131: 24-44

Simons M, Hager B (1997) Localization of the gravity field and the signature of glacial rebound. Nature 390: 500-504

Sun W, Okubo S (2004) Coseismic deformations detectable by satellite gravity missions: a case study of Alaska $(1964,2002)$ and Hokkaido (2003) earthquakes in the spectral domain. J Geophys Res 109:B04405. doi:10.1029/2003JB002554

Swenson S, Wahr J (2006) Post-processing removal of correlated errors in GRACE data. Geophy Res Lett 33:L08402. doi:10.1029/ 2005GL025285

Tamisiea ME, Mitrovica JX, Davis JL (2007) GRACE gravity data constrain ancient ice geometries and continental dynamics over Laurentia. Science 316: 881-883

Tapley BD, Bettadpur S, Watkins M, Reigber C (2004a) The gravity recovery and climate experiment: Mission overview and early results. Geophy Res Lett, Vol 31. doi:10.1029/2004GL019920

Tapley BD, Bettadpur S, Ries J, Thompson P, Watkins M (2004) GRACE measurements of mass variability in the earth system. Science 305:503-505

Wahr J, Swenson S, Velicogna I (2006) Accuracy of GRACE mass estimates. Geophys Res Lett 33:L06401. doi:10.1029/2005GL025305

Wieczorek MA (2007) The gravity and topography of the terrestrial planets. In: Spohn T (ed) Treatise on geophysics. vol 10. Planets and Moons. Elesevier, Amsterdam

Wieczorek MA, Simons FJ (2005) Localized spectral analysis on the sphere. Geophys J Int 162:655-675. doi:10.1111/j.1365-246X. 2005.02687.x

Wieczorek MA, Simons FJ (2007) Minimum-variance multitaper spectral estimation on the sphere. J Fourier Anal Appl (in press) 